\title{
Sociedad civil y cambio político y social en Argelia: evolución de discursos y estrategias
}

Civil society and socio-political changes in Algeria: evolution of discourses and strategies.

\section{Laurence THIEUX}

Universidad Complutense de Madrid

laurence.thieux@gmail.com

https://orcid.org/0000-0002-2532-1092

Recibido 6/11/2018. Revisado y aceptado para publicación 10/12/2018

Para citar este artículo: Laurence THIEUX (2018), "Sociedad civil y cambio político y social en Argelia: evolución de discursos y estrategias" en Revista de Estudios Internacionales Mediterráneos, 25, 79-92.

Para acceder a este artículo: https://doi.org/10.15366/reim2018.25.005

\section{Resumen}

La sociedad civil argelina tiene una larga trayectoria de movilización y contestación política y social que se remonta al final de los años 80, en un momento en el que Argelia iniciaba un proceso breve, pero intenso, de apertura política y pluralismo. La experiencia fue brutalmente abortada por el golpe llevado a cabo por los militares en. 1991. Desde entonces, la sociedad civil ha evolucionado tanto en sus discursos como en sus estrategias frente a un sistema político autocrático que ha permanecido inalterado a pesar de las olas de contestación política protagonizados por los movimientos sociales en el Norte de África y Oriente Medio a partir de 2011. El presente artículo pretende analizar la evolución de los principales discursos y estrategias de la sociedad civil y los movimientos sociales en Argelia que, a pesar de los factores inhibidores propios de la historia reciente de Argelia, como a la singularidad de su sistema político, son portadores de transformaciones sociales y de cambio político.

Palabras clave: Sociedad civil, movimientos sociales, protestas políticas, democracia, Argelia

\footnotetext{
${ }^{1}$ Este artículo forma parte de los resultados del proyecto I+D “Crisis y procesos de cambio regional en el norte de África. sus implicaciones para España” (CSO2017-84949-C3-3-P) financiado por el Ministerio de Economía, Industria y Competitividad (MINECO), la Agencia Estatal de Investigación (AEI) y el Fondo Europeo de Desarrollo Regional (FEDER).
} 


\begin{abstract}
Algerian civil society has a long history of mobilization and social and political resistance that dates to the end of the 1980s, when a brief but intense process of political openness and pluralism began. The incipient electoral process and the first signs of an autonomous sphere of social and political expression were brutally aborted by the military coup of 1991. Since then civil society has evolved in both its discourses and the strategies used against the autocratic political system, which has remained unchanged despite the waves of resistance led by social movements in North Africa and the Middle East since 2011. This article analyses the evolution of the main discourses and strategies used by Algerian civil society and social movements which, despite the inhibitory factors of the country's recent history and the uniqueness of its political system, are carriers of social transformations and political change.
\end{abstract}

Key words: Civil society, social movements, political protests, mobilization democracy, Algeria

\title{
Introducción
}

La evolución de los discursos y estrategias de la sociedad civil en Argelia ha estado profundamente marcada por el contexto político y social en el marco del cual ésta se ha gestado y ha dado sus primeros pasos. Una retrospectiva histórica, remontándonos a la década de los años 90 es por lo tanto fundamental para abordar la cuestión del discurso de la sociedad civil y su posicionamiento ante los grandes desafíos y retos nacionales frente a los cuales las organizaciones enmarcan sus acciones y estrategias.

El término sociedad civil abarca un amplio espectro de actores. En el presente artículo nos centraremos en el tejido asociativo y los sindicatos autónomos que han desempeñado un papel clave desde su creación a finales de los años 80. Para acotar aún más el objeto de estudio nos centraremos en las asociaciones que han tratado de crear un espacio autónomo (tanto a nivel de discurso como de acción) frente a la omnipresencia del Estado.

En las lógicas discursivas que no siempre encuentran su traducción en estrategias concretas, las asociaciones argelinas que emergieron a finales de los 80 en un nuevo entorno favorable creado por la liberalización política emprendida bajo la presidencia de Chadli Bendjedid han estado fuertemente condicionadas por la evolución del contexto político tras el golpe de estado militar de 1991. Sus discursos y estrategias han estado fuertemente constreñidas o condicionadas por la capacidad del régimen para coartar y/o cooptar las voces que no comulgan con la narrativa política dominante construida desde el poder.

En una primera parte se presentan los discursos y estrategias de las asociaciones que han protagonizado desde el final de los 80 la emergencia de la sociedad civil con el fin del monopartidismo y del discurso político único del Frente de Liberación Nacional (FLN) y otras organizaciones de masas como la Unión General de Trabajadores Argelinos (UGTA).

En la segunda parte del artículo se analiza su evolución así como el proceso de construcción de discursos políticos de sustitución, supliendo la ausencia de legitimidad de una oposición política debilitada y cooptada por el régimen. La clave de análisis que guiará nuestra reflexión es la relación mantenida con el poder que ha condicionado de forma explícita e implícita el posicionamiento de la sociedad civil y su actuación en sus ámbitos respectivos de influencia. 80 
Finalmente se analizarán las nuevas tendencias así como la diversificación de discursos que derivan de la exposición de la sociedad argelina a la globalización y la integración de nuevas temáticas en sus agendas de trabajo así como las manifestaciones de nuevas iniciativas ciudadanas tratando de liberarse del marco estrecho de maniobra en el cual evolucionó la generación anterior.

\section{La difícil emergencia de un discurso autónomo y crítico con el orden establecido}

Las transformaciones que las sociedades de la región MENA han experimentado en las dos últimas décadas han situado a la sociedad civil en el centro del discurso sobre los procesos de transición política. En efecto las organizaciones de la sociedad civil son percibidas como un agente clave de democratización, del pluralismo, expresión de una ciudadanía activa y como palanca fundamental para la sensibilización de la sociedad respecto a nuevos desafíos nacionales o globales.

La contribución de la sociedad civil a la democratización de las sociedades tiene que ser contrastada o evaluada a la luz de las experiencias concretas de las asociaciones sobre el terreno. En teoría para que una organización pueda desempeñar un papel de transformación y asumir funciones de promoción y difusión de la cultura de la democracia, de los derechos humanos, de denuncia y vigilancia de las prácticas gubernamentales así como su participación en la elaboración de políticas públicas son necesarias condiciones previas e ineludibles tales como la autonomía, la independencia de las autoridades, la capacidad de articulación y coordinación entre ellas y la integración de sus misiones en una agenda pro democrática (Hawthorn, 2013).

En relación con la agenda democrática, las organizaciones de la sociedad civil no siempre cumplen la función de ser agentes de cambios y contribuyen incluso, en algunos contextos, a consolidar y mantener los regímenes autoritarios (Cavatorta, 2012). En el caso de Argelia, la sociedad civil es también un actor clave de las estrategias de resiliencia diseñadas por el poder para mantener la estabilidad del sistema (Hamadouche, 2018).

Fue a partir de 1987 cuando tras la adopción de un marco legal menos rígido (ley no 87-15) comenzaron a ser creadas numerosas asociaciones como la Ligue des Droits de l'Homme (LADDH). Las revueltas populares de octubre de 1988 marcaron un punto de inflexión y aceleraron el ritmo de las reformas políticas y la apertura del espacio político. Durante este periodo el movimiento asociativo se construyó sobre la base de reivindicaciones para la promoción de un nuevo modelo de sociedad basado en los derechos fundamentales de los ciudadanos, la democracia, la reforma de las instituciones, el sistema educativo y la afirmación del pluralismo político, cultural y religioso.

En el contexto de apertura política de finales de los años 80 y con la aprobación de la Constitución de 1989 se abrió la vía a la modificación de la ley sobre las asociaciones lo que condujo a la adopción de la ley 90-31 de diciembre 1990 que consagró la libertad de asociación aliviando los trámites administrativos relativos a la creación de las asociaciones y al desarrollo de sus actividades. Esta liberalización del marco legal duró poco tiempo ya que con la instauración del estado de excepción (decreto no 92-44) volvieron a ser introducidas nuevas restricciones como la doble autorización, suprimida en la ley de 1990. Aun así durante estos años numerosas 
asociaciones vieron la luz. Entre 1990 y 1997, fueron creadas 57.000 asociaciones, 1.000 de ámbito nacional y 56.000 de ámbito local ${ }^{2}$ (Thieux, 2009).

Según datos del ministerio del interior en 2018, 91.102 asociaciones nacionales y locales estaban registradas en el ministerio. La mayor parte de las asociaciones argelinas concentran sus actividades en el ámbito social, cultural o en el medio ambiente, sectores en los cuales el Estado tiene interés en promover el tejido asociativo al considerar que su ámbito de actuación, en principio, no entra en conflicto con el Estado. Sin embargo, las asociaciones que basan su discurso en la defensa de los derechos humanos, la lucha contra la corrupción, la identidad bereber, la defensa de los derechos sindicales y de los trabajadores son minoritarias.

El fin del conflicto civil que asoló el país durante los años 90 y la normalización de la vida política tras el proceso de reconciliación nacional conducido por el Presidente Bouteflika tras su elección como presidente de la República en 1999 no aliviaron el peso de las restricciones sobre el espacio asociativo. La ambigüedad del marco jurídico y legal permitió a las autoridades continuar ejerciendo un control estrecho sobre la sociedad civil y, en particular, sobre las organizaciones más críticas con el régimen.

A principios de 2011 cuando las protestas antiautoritarias empezaron a desestabilizar los regímenes de Túnez y Egipto, Argelia también se encontraba en una situación delicada con un descontento popular manifestándose a través de multitudes de micro-revueltas localizadas en todos los rincones del país (11.500 registradas en el año 2010). Estas protestas siguen activas y en particular en el sur de Argelia (Hernando de Larramendi, y Thieux, 2018).

Entonces la fragmentación de la sociedad civil y la falta de credibilidad de los partidos políticos dificultaron la emergencia de estructuras organizadas capaces de orientar y dirigir un movimiento de contestación política de mayor calado. Si bien algunos actores de la sociedad civil lo intentaron a través de la plataforma Coordinación Nacional para el cambio y la democracia (CNCD) ${ }^{3}$, agrupando a distintos partidos y sindicatos, el intento no prosperó. La manifestación convocada en Argel en febrero de 2011 sólo consiguió congregar a unas 3.000 personas frente a un despliegue de 30.000 policías. La plataforma se escindió a las pocas semanas de su creación por las fuertes divergencias ideológicas existentes en su seno.

El peso de las restricciones sobre el espacio asociativo sigue obstaculizando el desarrollo de los proyectos asociativos y la ley de asociaciones $n^{\circ} 12-06$, aprobada por la Asamblea Nacional Popular el 13 de diciembre de 2011, introdujo disposiciones represivas tanto para la creación de nuevas asociaciones como para las ya existentes al someter a las asociaciones a la necesidad de renovar su autorización proceso que no está al alcance de las organizaciones que se sitúan en la oposición. La ley establece además mecanismos de control sobre la financiación externa de las asociaciones (Euromed Rights, 2012).

\footnotetext{
${ }^{2}$ De acuerdo con la plataforma Algeria Watch habría en Argelia 108.940 asociaciones registradas y la mayoría locales. Esta cifra no contempla las formas subterráneas o la acción colectiva ver : https://algeria-watch.org/?p=34191 [consulta 30/9/2018].

${ }^{3}$ La plataforma agrupaba a varias de las viejas figuras del sistema desde Sid Ahmed Ghozali (que era primer ministro durante la presidencia de Chadli Bendjedid en 1991), a Saïd Saadi de la Reagrupación para la Cultura y la Democracia RCD, un partido que apoyó el golpe de los generales que acabó con la experiencia de democratización de los años 90; la Liga Argelina de los Derechos Humanos (LADDH) junto con varios sindicatos autónomos.
}

82 
Mientras que la aplicación de la ley ha sido bastante flexible con las asociaciones con fines sociales y culturales que son ampliamente mayoritarias en Argelia, las más reivindicativas y entre ellas las que defienden los derechos humanos han tenido más dificultades para adaptarse a las disposiciones de la nueva ley. Asociaciones como el Rassemblement Action Jeunesse (RAJ) ${ }^{4}$ o la Ligue Algérienne des Droits de l'Homme ${ }^{5}$ (LADDH) no han conseguido obtener la cita obligatoria en el ministerio del interior para presentar sus nuevos estatutos. Muchas organizaciones se han visto obligadas a funcionar en la ilegalidad lo que ha fragilizado sus estructuras, limitando considerablemente sus capacidades de acción (imposibilidad de acceder a financiación de los donantes, o de alquilar una sala para realizar actividades y organizar encuentros).

Profundamente dividida tras la década de guerra civil que desgarró la sociedad argelina y su acción paralizada, el tejido asociativo empezó a recomponerse a partir de los años 2000. Se observa a partir de esta década una mayor implicación de las asociaciones en el sector de los servicios sociales, atendiendo a los colectivos más vulnerables (infancia, discapacitados...), Al mismo tiempo esta nueva generación de asociaciones se ha ido profesionalizando, un proceso respaldado por los actores internacionales (UE, Embajadas, Fundaciones y otras agencias de cooperación internacional) presentes en Argelia.

Como en otros contextos este proceso de profesionalización ha ido acompañado de un cambio de discurso con la adopción de modelos de trabajo y agendas en línea con las prioridades temáticas y sectoriales de las agendas de cooperación de los actores internacionales presentes en Argelia. Cabe citar a este respecto los programas de fortalecimiento de capacidades como ONG1 y ONG2 de la Unión Europea o el programa Joussour-Programme Concerté Pluri Acteur apoyado por Francia y otros actores como la cooperación belga, Embajada de Holanda, Canadá y Estados Unidos $^{6}$ (Kadri, 2012 y Roca, 2012).

\section{Expresión y margen de acción reducidos en un marco político autoritario}

Las características del entorno político y legal a los que nos hemos referido en el apartado anterior explican la predominancia de tres tipos de discursos y estrategias diferentes de la sociedad civil que ha evolucionado en compartimentos estancos y con pocas interacciones entre ellos, lo que constituye un claro obstáculo a la articulación de movimientos sociales de mayor alcance social y con propuestas y alternativas más sólidas frente al orden establecido.

Las fracturas y las divisiones entre los diferentes grupos de actores que conforman la sociedad civil argelina se articulan entorno a la posición que unos y otros mantienen frente al poder.

Surge esta polarización en la década de los 90 en relación con las estrategias adoptadas por los militares para eliminar del escenario político el Frente Islámico de Salvación (el partido islamista), vencedor de la primera vuelta de las elecciones legislativas de 2011 y muy probablemente de la segunda si el proceso no hubiera sido interrumpido por los militares en enero de 1992.

\footnotetext{
${ }^{4}$ https://arab.org/directory/rassemblement-actions-jeunesse/ [consulta 2/12/2018].

${ }^{5}$ https://laddh-algerie.org/?lang=fr [consulta 2/12/2018].

${ }^{6}$ Ver http://www.pcpalgerie.org/ [consulta 30/10/2018]
} 
En ese momento, parte de las organizaciones (que acababan de iniciar su andadura) renunciarían a la defensa de la democracia apoyando el golpe de los generales. Es el caso de algunas organizaciones de mujeres que se alinearán con el régimen frente a los islamistas como el "Rassemblement Algérien des Femmes Démocrates" de Zazi Sadou y de Khalida Messaoudi. Los generales buscaban entonces el apoyo de la sociedad civil y las fuerzas políticas opuestas al proyecto islamista. El “Comité National de la Sauvegarde de l'Algérie" impulsado por el gobierno y liderado por Abdelhak Benhamouda de la UGTA servirá de enlace en el seno de la sociedad civil para fortalecer la legitimidad del discurso erradicador de las fuerzas islamistas.

La interrupción de las elecciones en enero de 1991 por los generales desembocó en una cruenta guerra civil creando profundas heridas y divisiones en la sociedad argelina que aún perviven en la actualidad.

Estas divisiones se acentuaron aún más, atenazadas en la espiral de violencia entre militares y grupos armados. En ese contexto las organizaciones de la sociedad covil (OSC) se dividieron aún más entre "erradicadores" y "partidarios del diálogo".

La década negra con sus consecuencias dramáticas sobre la población civil (200.000 muertos y 150.000 desaparecidos) tuvo consecuencias muy profundas y duraderas sobre la sociedad argelina cuyos efectos son aún palpables hoy en día. La destrucción de los vínculos sociales, la desconfianza, la ausencia de un verdadero proceso de reconciliación y de verdad han tenido un impacto negativo sobre la sociedad civil.

Casi 20 años después, estas líneas de fractura persisten entorno a la posición de las organizaciones respecto al poder. Así cabe distinguir tres grandes tendencias que orientan el posicionamiento y actuación de las organizaciones de la sociedad civil en Argelia.

Tanto en los discursos como en las estrategias, la relación con el Estado constituye por lo tanto un claro polo de divergencia en el seno de la sociedad civil argelina pudiendo distinguirse entre las organizaciones que dócilmente se someten a los procesos de cooptación o satelización y otras se sitúan en el lado opuesto de la confrontación.

Un primer núcleo de organizaciones mantiene una oposición firme al sistema político y ha desarrollado un discurso crítico respecto a la legitimidad del gobierno, su actuación en materia de derechos humanos y libertades públicas así como hacia la corrupción y otras malversaciones de los círculos que han monopolizado el poder desde la independencia. Este discurso de oposición tiene un alcance limitado. Las organizaciones que se sitúan en esta tendencia (algunas organizaciones de derechos humanos como la Liga Argelina de Derechos Humanos (LADDH), algunas asociaciones de defensa de los derechos de las mujeres, el Reagrupamiento Acción Juventud (RAJ), I'a Asociación Argelina de Lucha contra la Corrupción $(A A C C)^{7}$, el Colectivo de las Familias de Desaparecidos $(\text { CFDA })^{8}$, SOS Desaparecidos ${ }^{9}$ tienen un margen de maniobra limitado y han evolucionado en autarquía para mantenerse a salvo de las sofisticadas tácticas que el gobierno utilizar para neutralizar las voces que cuestionan su monopolio sobre el poder político. Esta primera corriente

\footnotetext{
${ }^{7}$ https://www.facebook.com/pages/category/Non-Governmental-Organization--NGO-/AACC-AssociationAlg\%C3\%A9rienne-de-lutte-contre-la-corruption-215181501888412/ [consulta 2/12/2018].

${ }^{8}$ http://www.algerie-disparus.org/ [consulta 2/12/2018].

${ }^{9}$ https://www.facebook.com/disparusalgerie/ [consulta 2/12/2018].

84
} 
que abarca organizaciones que nacieron a finales de los 80 y otras que surgieron después en la década de los 90 está profundamente marcada por la polarización ideológica que dominó estas dos décadas en el contexto de la lucha del régimen de los militares contra los grupos armados islamistas.

Una mención particular merece el discurso y las estrategias de los sindicatos autónomos que han conseguido combinar cierto apoyo local y resonancia internacional para consolidar un espacio de expresión e influencia autónomos aunque limitado ${ }^{10}$.

Hasta la Constitución de 1989 Argelia solo contaba con un sindicato: la Unión General de los Trabajadores Argelinos (UGTA) ${ }^{11}$. La Ley 90-14 establece un régimen de registro que obliga a la obtención de un certificado de actividad por parte de la administración cuando se deposita la solicitud de creación. Sin este certificado de actividad la organización no puede funcionar y su retención queda al arbitrio de una decisión negativa de la administración. Esta práctica está denunciada por las organizaciones sindicales ya que contraviene el convenio sobre la libertad sindical y la protección del derecho de sindicación, 1948 (núm. 87), ratificado por Argelia en 1962 al indicar que solo una instancia judicial está habilitada para establecer la conformidad de un proyecto de creación de un sindicato. Desde los años 90 numerosas organizaciones sindicales no han obtenido su autorización.

Los sindicatos autónomos han desarrollado sus actividades en un entorno hostil marcado por la ausencia de diálogo con las instituciones. Varios sindicatos han sido creados como el Sindicato Autónomo del Personal de la Administración Pública (SNAPAP) ${ }^{12}$, el Sindicato Nacional de la Enseñanza Superior (SNES), el Sindicato Autónomo de los Trabajadores de la educación y de la formación (SATEF) ${ }^{13}$, el Consejo Nacional Autónomo de los Profesores de la enseñanza segundaria $y$ técnica $\left(\right.$ CNAPEST) ${ }^{14}$.

Estos sindicatos constituyen un polo de movilización y oposición importante. Han sido objeto de represión y otros intentos de manipulación o clonación a través de la creación de entidades similares. Con estas prácticas de injerencia en la actividad sindical así como la clonación de las siglas de diferentes organizaciones, se trata de generar la duda sobre la representatividad de las organizaciones autónomas provocando la confusión no solo en el ámbito nacional argelino sino también en el internacional. A pesar de las presiones los sindicatos han tratado de reforzar los vínculos entre ellos al crear una confederación, por primera vez, como alternativa a la histórica UGTA: la Confederación General Autónoma de Trabajadores de Argelia (CGATA) ${ }^{15}$, intersindical

10 Ver al respecto: Individual Case (CAS) - Discussion: 2018, Publication: 107th ILC session (2018) https://www.ilo.org/dyn/normlex/en/f?p=1000:13100:0::NO:13100:P13100 COMMENT ID:3953276 [consulta $1 / 12 / 2018]$.

${ }^{11}$ http://www.sneu-ugta.dz/ [consulta 2/12/2018].

12 http://www.snapap.dz/ [consulta 2/12/2018].

13 http://satefalgerie.eklablog.com/ [consulta 2/12/2018].

${ }^{14}$ https://www.cnapeste.dz/ [consulta 2/12/2018].

${ }^{15}$ https://www.facebook.com/pages/category/Non-Governmental-Organization--NGO-/CGATAConf\%C3\%A9d\%C3\%A9ration-g\%C3\%A9n\%C3\%A9rale-autonome-des-travailleurs-en-Alg\%C3\%A9rie750457288343416/ [consulta 2/12/2018]. 
autónoma de la función pública creada en 2008 y que reúne a varias formaciones (SNAPAP, CLA, CNAPEST, SATEF, SNTE, SNPEPM, CECA) que ha conseguido su afiliación internacional a la Confederación Sindical Internacional (CSI).

Tanto las organizaciones de derechos humanos como los sindicatos autónomos han desarrollado un discurso de oposición intransigente con el poder: la naturaleza del régimen, su carácter autoritario y su voluntad de control de la sociedad son incompatibles con la autonomía de la sociedad civil y cualquier vínculo termina siendo instrumentalizado o utilizado como una vía de cooptación.

Estas organizaciones han tratado a través de su discurso romper la narrativa del poder y se encuentran por lo tanto bajo el foco de la acción represiva del Estado.

Cabe citar como ejemplo de ruptura con el discurso oficial del colectivo de las familias de los desaparecidos en Argelia (CFDA) - SOS desaparecidos/as respecto al proceso de reconciliación nacional impulsado por Buteflika a través de la Concordia civil en 1999 y la Carta de 2005 que cierra la puerta a procesos de reconciliación basados en la verdad y la memoria. Son precisamente estas asociaciones las que ven vetadas su acceso a la legalidad. La misma suerte tiene la Asociación Argelina de lucha contra la corrupción tras el intento de obtener una autorización en octubre de 2012 (Human Rights Watch: 2014).

Estas organizaciones tratan de mantenerse fuera del alcance de las injerencias del poder para conservar su autonomía y mantienen escasas relaciones con las autoridades: el RAJ, por ejemplo, no mantiene ninguna relación con los ministerios a excepción de algunos contactos puntuales con instituciones intermedias como la dirección de la juventud y los deportes o la dirección de acción social. SOS desaparecidos, estableció algunos contactos con el ministerio de justicia a principios de los años 2000 en el momento de la creación de la Comisión Ksentini y la creación de un mecanismo ad-hoc para ocuparse del expediente de los desaparecidos (2003) pero pronto se distanció de este mecanismo oficial incapaz de responder a las demandas de las familias por falta de capacidad y voluntad política.

Frente a esta primera corriente de sociedad civil que se sitúa en la oposición frontal al régimen, encontramos otras organizaciones que han ido ocupando el espacio dejado vacío por las instituciones en el ámbito social sin interferir ni cuestionar la gestión política del Estado. Las organizaciones que se ubican en esta tendencia, se han desarrollado al amparo de las autoridades que perciben a estos actores como un apoyo claro al mantenimiento del sistema. En su discurso algunas de estas organizaciones defienden sus vínculos con la administración y las instituciones como la mejor vía de introducir cambios significativos y mejoras en términos de transparencia y participación a través de la colaboración. La red NADA ${ }^{16}$, que trabaja en el ámbito de la infancia, se situaría claramente en esta tendencia que es percibida por las organizaciones que se ubican en la confrontación como satélites y/o prolongación del Estado.

Se tratan de dos lógicas muy diferentes basadas sobre un análisis del poder y de las palancas a utilizar para promover el cambio radicalmente opuestas.

\footnotetext{
${ }^{16}$ http://www.reseau-nada.org/ [consulta 2/12/2018]
} 
Dentro de esta tendencia algunas organizaciones están directamente vinculadas con el poder y son instrumentalizadas para fines políticos concretos, como en el momento de las elecciones, para apoyar el candidato presidencial o defender una causa concreta. La instrumentalización del tejido asociativo "oficial" en el Foro Social Mundial de 2015 celebrado en Túnez es un ejemplo claro. El gobierno facilitó la presencia masiva de miembros de la sociedad civil (40 autobuses) para garantizar la representación de organizaciones afines al poder para contrarrestar a las organizaciones marroquíes que iban a defender la postura de Marruecos sobre la cuestión del Sahara Occidental (Rondeleux, 2015).

Dentro del amplio abanico de asociaciones que han optado por la colaboración y diálogo con las instituciones existen también estrategias variadas y distintos grados de vinculación con el poder.

En el ámbito de la protección de la infancia, o la lucha contra la violencia de las mujeres o de la asistencia a colectivos vulnerables como por ejemplo las personas con discapacidad, las asociaciones tratan a través de los vínculos establecidos con las instituciones y autoridades locales o nacionales de ejercer presiones para impulsar un cambio en las políticas públicas. Cuentan además con una experiencia y pericia en sus respectivos ámbitos de actuación que constituyen un valor añadido importante a la hora de formular propuestas para mejorar prácticas, procedimientos y marcos legales y ejercer, a través de esta vía, una influencia sobre los responsables del diseño y aplicación de las políticas públicas. Es el caso por ejemplo de la Federación Argelina de personas discapacitadas (FAPH) ${ }^{17}$, que después de haber integrado los espacios de concertación creados por el ministerio de solidaridad para mejorar la accesibilidad de este colectivo a los servicios públicos, transporte y constatar su ineficiencia ha impulsado la formación de una comisión paralela reuniendo diferentes actores como sindicatos, colectivos de abogados, gabinetes de estudios para plantear propuestas concretas .

Las experiencias de colaboración y participación de las asociaciones a la concepción y realización de políticas públicas se dan más a nivel local y en sectores como la atención a los colectivos más vulnerables, el medio ambiente, la salud, la inserción económica o la educación (Benramdane, 2015). La experiencia de la Asociación Chougrani ${ }^{18}$ en Orán es otro ejemplo de cómo las asociaciones combinan la colaboración con la administración con la realización paralela de presiones sobre las autoridades competentes. En el caso de esta asociación local el objetivo era obtener el reconocimiento de profesiones emergentes en el ámbito de la salud pública (como el cuidado de las personas mayores y las personas con discapacidades).

Finalmente cabe identificar un tercer sector portador de un discurso emergente desde la periferia y canalizando las frustraciones originadas por la acumulación de marginalidades (geográfica, económica, social y política) que viene a cuestionar indirectamente la legitimidad y gestión política de las autoridades sin entrar directamente en cuestiones políticas.

En las regiones del sur de Argelia, y en respuesta a la degradación o de la situación económica y social de algunos sectores de la población, aparecieron nuevos movimientos como los diplomados desempleados. El Comité nacional para la Defensa de los Derechos de los Desempleados

\footnotetext{
${ }^{17}$ https://faphblog.wordpress.com/ [consulta 2/12/2018].

18 https://www.facebook.com/groups/APROSCH/about/ [consulta 2/12/2018].
} 
$(C N D D C)^{19}$ ha tenido un papel clave en poner de manifiesto el profundo malestar de la población de las provincias del Sur del país y en particular de los jóvenes sin empleo.

Los movimientos de protestas que han surgido en los últimos años en localidades como Ouargla, Hassi Messaoud o In Salah, regiones ricas en recursos petrolíferos y yacimientos de gas ponen de manifiesto el fracaso de las políticas de desarrollo en las provincias del Sur del país que acusan particularmente el desempleo, la falta de estructuras sociales y las desigualdades económicas y la redistribución insatisfactoria de sus dividendos entre la población (Hernando de Larramendi y Thieux, 2018).

Existe por parte de los que han protagonizado estas protestas una voluntad de mantener su autonomía e independencia en relación con otras organizaciones de la sociedad civil. Han podido recibir apoyos ${ }^{20}$ pero sin establecer lazos más vinculantes.

Estas reticencias se explican también por la necesidad de contrarrestar los intentos de manipulación, infiltración que el Estado utiliza para neutralizar estos movimientos de protestas. Cabe resaltar al respecto el ejemplo de las protestas de In Salah de 2015 contra la explotación del gas de esquisto y sus efectos contaminantes y muy dañinos para la población local. De las protestas surgió un colectivo Nacional del No al gas de Esquisto pero conservando su carácter local para evitar posibles infiltraciones. El temor a ser instrumentalizado ha motivado su rechazo en participar en diversas plataformas o foros como el Foro Social Mundial pudieran diluir sus reivindicaciones (Mañé, Thieux y Hernando de Larramendi, 2016).

Una vez más se puede observar cómo las sofisticadas estrategias desplegadas por el poder para neutralizar las contestaciones al orden establecido han condicionado el discurso y las estrategias de la sociedad civil y los movimientos sociales.

Estos movimientos de protestas recurrentes, por diferentes motivos (colapso del alcantarillado, mal estado de los hospitales....) cuyas manifestaciones más recientes protagonizaron los habitantes de Ouargla en verano de 2018 (Alioua, 2018), vienen a cuestionar el sistema de gobernanza del estado, la corrupción y el sistema autoritario poniendo en cuestión el contrato social post-colonial que consistía en que el pueblo renunciara a parte de las libertades políticas ciudadanas a cambio de derechos sociales (Hernando de Larramendi y Thieux, 2018).

Las tensiones identitarias han sido otra de las manifestaciones de este profundo malestar social. En la región del M'zab, los enfrentamientos entre la minoría ibadita (mozabitas) y otras comunidades son también consecuencia de la ruptura de los equilibrios socio-económicos producidos por el cierre de las fronteras por la inestabilidad de Libia y la repercusión sobre las actividades económicas transfronterizas que constituyen el medio de vida de una parte importante de la población. La zona del M’zab se ha visto afectada en los últimos años por estas

\footnotetext{
${ }^{19}$ Fue creado en el 2011 y adquirió visibilidad nacional a partir de 2013 con la manifestación de los 8.000, conocida como la marcha del millón, en la que se protestaba contra el desempleo, la hogra y la orientación neoliberal de la economía. En poco tiempo logró articular un movimiento pacífico y canalizar las frustraciones de muchos colectivos, entre ellos el de los jóvenes desempleados. Para más información ver (Mañé Thieux y Hernando de Larramendi 2016).

${ }^{20}$ En el caso del CNDDC recibió el respaldo de otras organizaciones de la sociedad civil como el Sindicato Nacional Autónomo del Personal de la Administración Pública (SNAPAP), el de la Red de Abogados de los Derechos Humanos (RADDH), organización que asumió su defensa en los procesos judiciales, y el de la Liga Argelina de los Derechos Humanos (LADDH).
} 
transformaciones que han desembocado en un proceso de inmigración interna afectando particularmente algunas ciudades como Gardaia o Beni Isguen (Benantar, 2016).

La ineficacia de las políticas públicas frente a los numerosos problemas de desarrollo a los que estas regiones se ven confrontadas está en el centro del discurso y de las movilizaciones de los actores locales. Las asociaciones juegan un papel clave para encuadrar las reivindicaciones y tratar de mediar con las autoridades.

Estas tres tendencias o corrientes principales han evolucionado de forma separada, con bajos niveles de interacción entre ellas. Asimismo el impacto que estas tres tendencias han podido tener sobre el proceso de apertura política es variable y contrastado.

La relación mantenida con el poder es un elemento central que condiciona de forma determinante el discurso y las estrategias de las asociaciones. Aun así cabe recalcar o resulta sorprendente notar que frente a la adopción de la ley de 2012 que establece un marco restrictivo para las asociaciones, estas últimas no se hayan movilizado de forma clara al respecto ${ }^{21}$.

En el contexto argelino cabe recordar que el papel democratizador de la sociedad civil está muy condicionado por las estrategias utilizadas por los que tienen las riendas del poder. Estos últimos tratan de contrarrestar y neutralizar las estrategias percibidas como más sediciosas e intentan a la vez cooptar y sellar alianzas con otro sector de la sociedad civil con el fin de capitalizar la legitimidad que esta última puede brindar al poder escondiendo su carácter autoritario bajo un pluralismo de fachada. En el caso argelino las estrategias utilizadas por el poder para neutralizar las expresiones autónomas de la sociedad civil han sido variadas y sofisticadas. Cabe citar las siguientes:

La represión destinada a neutralizar la capacidad de oposición de las organizaciones de la sociedad civil reprimiendo su libertad de acción mediante la adopción de leyes o trabas administrativas.

El despliegue de tácticas más sofisticadas como la clonación (ejemplo sindicatos autónomos) o la infiltración de ciertas organizaciones,

- La utilización de las asociaciones como paliativos a la retirada del Estado en determinadas prestaciones sociales.

- La creación de una sociedad civil a medida con la emergencia de asociaciones satélites del poder y movilizadas para apoyar el régimen en momentos claves como las elecciones $u$ otros acontecimientos y destinadas a mejorar la imagen de Argelia en el exterior (Foro Social de Túnez) (Desrues y Velasco Arranz, 2015).

En contextos políticos autoritarios la sociedad civil pasa a ser en muchos casos un espacio de sustitución para la expresión de proyectos políticos de oposición frente a la debilidad de los partidos políticos integrados al sistema o reprimidos.

\footnotetext{
${ }^{21}$ Entrevistas realizadas en Argel y Blida del 28 de noviembre al 4 de diciembre de 2016 con actores de la sociedad civil argelina en el marco de una investigación para Alianza para la Solidaridad financiada por la Union Europea «Decent work and social protection and freedom of association in the Middle East and North Africa: mobilizing for social justice by strengthening and promoting CSOs, social movement and (independent) trade union's role in reform and democratic changes » (ENPI/2014/353-573).
} 
Existe además un trasvase bastante habitual entre los dos ámbitos: personalidades políticas que desarrollan un proyecto asociativo o figuras del mundo asociativo que se presentan como candidatos en los procesos electorales. En algunos casos las asociaciones están fuertemente vinculadas a un partido político. Los vínculos mantenidos entre el partido político Frente de Fuerzas Socialistas (FFS) y asociaciones como la LADDH o el movimiento RAJ son tan sólo un ejemplo ilustrando la fuerte imbricación entre movimientos asociativos y partidos políticos con una influencia clara en las líneas de discurso desarrolladas por estas organizaciones.

La vinculación ideológica de las asociaciones con los partidos políticos tiene a su vez consecuencias negativas sobre la capacidad de las organizaciones de extender su base o influencia y trascender la esfera de influencia del partido político de referencia.

De lo anterior también deriva otra consecuencia negativa observable en el terreno: la dificultad de la sociedad civil para iniciar un trabajo inter asociativo que le permitiría ensanchar su base social y capacidad de movilización, una de las claves de éxito de los movimientos de protestas de Túnez que acabó con el régimen de Ben Ali.

Las divisiones y el clima generalizado de sospecha dificultan los procesos de articulación y coordinación entre las organizaciones de la sociedad civil, el trabajo en red o la formación de coaliciones puntuales o plataformas sobre ciertas temáticas.

Finalmente el potencial de transformación de las organizaciones de la sociedad civil en Argelia también queda mermado por la permanencia de una cultura asociativa dominada por el presidencialismo y el culto al zaim. Así, algunas organizaciones contribuyen a reforzar y no debilitar la cultura autoritaria al reproducir en parte modelos organizativos muy jerarquizados $y$ poco democráticos y transparentes (Pratt, 2007).

En efecto, las asociaciones pueden ser portadoras de valores democráticos no sólo por sus mensajes (sensibilización, incidencia política) pero también por sus prácticas a través de los diferentes modelos de organización internos que se pueden evaluar en función de criterios claves como la transparencia, la participación o la gobernanza democrática de los órganos de gestión de las organizaciones.

No es el caso de todas las asociaciones que en algunos casos reproducen en su seno las mismas prácticas autoritarias que se encuentran en otras estructuras de la sociedad o ámbitos políticos y económicos (presidencialismo, mecanismos de toma de decisión verticales y jerárquicos, ausencia de mujeres y jóvenes en los puestos de responsabilidad).

\section{Conclusión}

La relación con la esfera del poder constituye uno de los ejes centrales que estructura el discurso de la sociedad civil argelina: desde el alineamiento hasta la búsqueda de espacios de autonomía, las diferentes estrategias desplegadas por el tejido asociativo tratan de adaptarse a través de diferentes respuestas a las restricciones impuestas por los gobernantes para contrarrestar la emergencia de un discurso contra hegemónico susceptible de cuestionar su monopolio de poder.

Las asociaciones que se sitúan en la confrontación son las que están más expuestas a la represión y tienen por lo tanto un margen de maniobra muy estrecho. Otras organizaciones trabajando en 
distintos sectores de la acción social buscan vías de colaboración con las instituciones como la mejor forma de alcanzar sus objetivos. Estas últimas, al aceptar el diálogo y la concertación con las autoridades contribuyen también al legitimar el poder establecido.

Tanto la confrontación como la sumisión al juego de poder son estrategias que ponen de manifiesto y alimentan la debilidad y fragmentación de la sociedad civil quedando así anulado o reducido al mínimo el papel de mediación entre las demandas que emanan de diferentes sectores de la sociedad y el Estado. La ausencia de estas vías tiene como consecuencia principal la multiplicación de micro-revueltas a las cuales el Estado rentista y redistribuir trata de ahogar respondiendo parcialmente a las demandas formuladas.

Son las desigualdades horizontales afectando a sectores de la población en las regiones de Sur las que han originado nuevas formas de movilización ciudadana (Mottaghi, 2018). Las nuevas tecnologías y las redes sociales, como Facebook se han convertido en un espacio privilegiado de concertación debate y movilización de los ciudadanos ${ }^{22}$, una forma de escapar también a las trabas legales y administrativas que ahogan los proyectos asociativos.

\section{Bibliografía}

ALIOUA, Houria (2018): "Vive tension à Ouargla". El Watan, disponible en https://www.elwatan.com/a-la-une/vive-tension-a-ouargla-15-09-2018 [consulta: 15/9/2018].

BENANTAR, Abdennour (2016): "Sécurité aux frontières: Portée et limites de la stratégie algérienne", L'Année du Maghreb, no 14, pp. 147-163. https://doi.org/10.4000/anneemaghreb.2712.

BENRAMDANE, Djamel (2015) : Les associations algériennes, des acteurs émergents en quête de reconnaissance. Informe del Comitato Internazionale per lo Sviluppo dei Popoli (CISP)

CAVATORTA, Francesco (2012): "Arab Spring: the awakening of civil society. A general overview" en Anuario IEMED 2012. Disponible en http://www.iemed.org/publicacions/historic-depublicacions/anuari-de-la-mediterrania/sumaris/anuari-iemed-de-la-mediterrania-2012-es

[consulta: 25/9/2018].

CERROLAZA, Erika (2013): "La sociedad civil argelina y la primavera árabe", en Sociedad civil y transiciones en el Norte de África, Barcelona, Icaria, pp. 71-128. Disponible en http://www.icariaeditorial.com/pdf libros/sociedad\%20civil\%20norte\%20Africa.pdf $\quad$ [consulta: 25/9/2018].

DESRUES, Thierry y VELASCO ARRANZ, Ana (2015): "Jóvenes y activismo político y social en el Magreb: los participantes en el Foro social mundial de Túnez en 2013", Revista de Estudios Internacionales Mediterráneos, no 15. http://dx.doi.org/10.15366/reim2015.19.002

HERNANDO DE LARRAMENDI, Miguel y THIEUX, Laurence (2018): "Protestas en la periferia. Contestación y desequilibrios en el Magreb", Notes Internationals 203, CIDOB, https://www.cidob.org/es/publicaciones/serie de publicacion/notes internacionals/n1 203/prot estas en la_periferia_contestacion_y desequilibrios_en_el magreb [consulta: 1/12/2018].

\footnotetext{
${ }^{22}$ Argelia contaría con 17 millones de usuarios de Facebook 43\% de la población, según datos aportados African digital Summit 2017 de Casablanca, ver https://www.algerie1.com/index.php/tech/17-millionsdutilisateurs-facebook-en-algerie [consulta 25/10/2018].
} 
DRIS-AÏT HAMADOUCHE, Louisa (2018): “Au cœur de la résilience algérienne: un jeu calculé d'alliances". Confluences Méditerranée, no 3, pp. 195-210. https://doi.org/10.3917/come.106.0195

HAWTHORNE, Amy (2013): "Is civil society the answer", Carnegie Papers, 2004, Disponible en http://carnegieendowment.org/files/CarnegiePaper44.pdf [consulta: 13/8/2018].

HUMAN RIGHTS WATCH (2014): "Algérie: Recours à des stratagèmes bureaucratiques pour entraver le travail des associations $"$, disponible en https://www.hrw.org/fr/news/2014/03/31/algerie-recours-des-stratagemes-bureaucratiquespour-entraver-le-travail-des [consulta : 16/9/2018].

EUROMEDITERRANEAN HUMAN RIGHTS NETWORK (2012): "Réformes politiques» ou verrouillage supplémentaire de la société civile et du champ politique ?" disponible en https://www.ldhfrance.org/Reformes-politiques-ou/ [consulta : 14/9/2018]

KADRI, Aïssa (2012) : “Associations et ONG au Maghreb", NAQD, no 29, pp. 87-117. Disponible en https://www.cairn.info/article.php?ID ARTICLE=NAQD 0290087 [consulta: 16/9/2018].

MAÑÉ, Aurelia, THIEUX, Laurence y HERNANDO DE LARRAMENDI, Miguel (2016): “Argelia en la encrucijada: condicionantes, tendencias y escenarios", Documento de Trabajo Opex, no 82/2016. Disponible

http://www.fundacionalternativas.org/public/storage/recursos descargas/bbcfd95a94692908df3 57bc38cbd45e3.pdf [consulta: 1/9/2018].

MOTTAGHI, Lili (2018): "Desigualdades y conflictos en la región MENA", Afkar Ideas, no 56, pp. 5457. https://www.iemed.org/observatori/arees-danalisi/arxiusadjunts/afkar/afkar56/esp/19Lili\%20Mottaghi\%20.pdf [consulta : 14/9/2018].

ROCA, Pierre Jean (2012): "Les associations «modernes», fer de lance de la société civile?" Confluences Méditerranée, no 2, pp. 69-81. https://doi.org/10.3917/come.081.0069

RONDELEUX, Nejma (2015) : "Des militants de la "vraie" société civile algérienne dénoncent les agissements de la délégation officielle au FSM Tunis" disponible en https://www.huffpostmaghreb.com/2015/03/27/fsm-tunisie-agression n 6955512.html [consulta: 15/9/2018].

PRATT, Nicola Christine (2007): Democracy and authoritarianism in the Arab world, Londres, Boulder.

THIEUX, Laurence (2014): "Sociedad civil, movilización colectiva y cambios políticos en el Norte de África” en Anuario CEIPAZ, no 7, pp. 113-138. Disponible en http://www.ceipaz.org/images/contenido/6.LauranceThieux.pdf [consulta 30/8/2018].

THIEUX, Laurence (2009) : "Le secteur associatif en Algérie: la difficile émergence d'un espace de contestation politique", L'Année du Maghreb, V, pp. 129-144. https://doi.org/10.4000/anneemaghreb.545 